\title{
Energy Analysis and Verification of a Constant Pressure Elastic Strain Energy Accumulator Based on Exergy Method
}

Hongwang Du ( $\square$ duhw_1984@dlmu.edu.cn )

Dalian Maritime University https://orcid.org/0000-0001-7974-9804

Dongdong Yang

Dalian Maritime University

Wei Xiong

Dalian Maritime University

Original Article

Keywords: Strain energy accumulator, Constant pressure energy supply, Energy storage efficiency, Exergy analysis, Mullins effect

Posted Date: January 23rd, 2021

DOl: https://doi.org/10.21203/rs.3.rs-151158/v1

License: (c) (i) This work is licensed under a Creative Commons Attribution 4.0 International License. Read Full License 


\section{Title page}

\section{Energy Analysis and Verification of a Constant Pressure Elastic Strain Energy Accumulator Based on Exergy Method}

Hong-Wang Du, born in 1984, is currently a associate professor and MD candidate supervisor at Naval Architecture and Marine Engineering School, Dalian Maritime University, China. His main research interests include fluid power transmission, virtual assembly technology and marine salvage technology.

Tel: +86-0411-84723725; E-mail: duhw_1984@dlmu.edu.cn

Dong-dong Yang, born in 1993, is currently a master candidate at Naval Architecture and Marine Engineering School, Dalian Maritime University, China.

E-mail: yd17516067775@163.com

Wei Xiong, born in 1972, is currently a professor and $\mathrm{PhD}$ candidate supervisor at Naval Architecture and Marine Engineering School, Dalian Maritime University, China. His main research interests include fluid power transmission, virtual assembly technology and marine salvage engineering equipment.

Tel: +86-0411- 84727060; E-mail: xiongwei@ dlmu.edu.cn

Corresponding author: Hong-Wang Du E-mail: duhw_1984@dlmu.edu.cn 


\title{
Energy Efficiency Analysis and Verification of a Constant Pressure Elastic Strain Energy Accumulator Based on Exergy Method
}

\author{
Hong-Wang Du ${ }^{1} \cdot$ Dong-dong Yang ${ }^{1} \bullet$ Wei Xiong ${ }^{1}$
}

\begin{abstract}
Aiming at problems of low energy storage efficiency and unstable energy output of existing accumulators, this paper proposes a novel constant pressure elastic strain energy accumulator based on the rubber material hyperelastic effect, which can store and release energy with steady constant pressure. Based on exergy analysis method, constant pressure elastic strain energy accumulator charging/discharging energy storage efficiency is analyzed. Then Mullins effect on the rubber airbag multiple charging/discharging cycles is studied. Finally, a test platform is set up to verify the energy storage efficiency, expansion and contraction pressure stability of the rubber accumulator during charging/discharging cycles. Compared with enthalpy analysis method, experiment results show that energy storage efficiency calculation by the exergy analysis method is more accurate. In more than 200 cycle tests, rubber airbag energy storage efficiency is always higher than $76 \%$, and expansion pressure and contraction pressure errors under steady state are less than $2.92 \mathrm{e}-3 \mathrm{MPa}$ and $1.79 \mathrm{e}-3 \mathrm{MPa}$, respectively. The results show that the rubber airbag can be used as an effective energy storage component, which is very meaningful for energy recovery in pneumatic or hydraulic systems.
\end{abstract}

Keywords: Strain energy accumulator $\bullet$ Constant pressure energy supply $\bullet$ Energy storage efficiency $\bullet$ Exergy analysis $\bullet$ Mullins effect

Hong-Wang Du

duhw_1984@dlmu.edu.cn

Naval Architecture and Marine Engineering School, Dalian Maritime University, Dalian 116026, China

\section{Introduction}

As common energy storage elements, accumulators are often used in hydraulic systems for energy recovery. Among them, the airbag type hydraulic accumulator is often used as an energy storage device in a hydraulic hybrid system to recover the energy generated when the car is braked and supply power when the car is restarted [1]. Studies have shown that the hydraulic hybrid technology has been applied to vehicles, fuel savings of $12-25 \%$ can be achieved in urban areas and fuel savings of $6-10 \%$ can be achieved during long-distance driving [2-3]. However, because the airbag type hydraulic accumulator cannot store and release energy at a constant pressure, the energy is often not fully recovered and released due to the mismatch with the system pressure during the working process, resulting in energy waste [4]. Although the airbag accumulator is used as an auxiliary energy source and its power density meets use requirements, the volume energy density and weight energy density are obviously not enough [5], which make it difficult to apply hydraulic hybrid technology to small cars.

Many scholars have conducted researches on hydraulic hybrid power systems and energy storage element accumulators. Qingyong Zhang [6] carried out theoretical analysis on the airbag type hydraulic energy storage of hydraulically driven hybrid vehicles; the results showed that under the same initial braking pressure, the pressure 
growth rate and pressure change range in the chamber were smaller when the accumulator volume was larger. Under the requirement of vehicle braking performance, reducing the accumulator volume and charging pressure increased the specific energy of the energy storage element. Bo Wang et al. [7] proposed a new configuration of hydraulic hybrid electric vehicle based on compound accumulators. Simulation results showed that the hydraulic hybrid electric vehicle based on compound accumulators can switch the working timing of large and small accumulators to take into account the energy recovery rate and braking performance, when the braking deceleration was $1.5 \mathrm{~m} / \mathrm{s}^{2}$, the energy recovery rate was $14.5 \%$ higher than that of a single accumulator; Yunsong $\mathrm{Lu}$ [5] presented that the use of two-phase media could stabilize the output pressure of the accumulator and increase the energy density of the accumulator by 3 to 5 times, the difficulty was that the saturated vapor pressure was difficult to meet the ideal requirements. Yunsong $\mathrm{Lu}$ raised a cylinder accumulator using a multi-layer structure of carbon fiber and high-strength metal. When the working pressure was $100 \mathrm{MPa}$, the energy density and specific energy were $105 \mathrm{~J} / \mathrm{L}$ and $14 \mathrm{Wh} / \mathrm{kg}$, which were 10 and 20 times than existed rigid accumulators, but it still did not solved the problem of constant pressure energy storage and release, and the production cost was higher. Alexander P and Eric J. Barth [8] put forward a hydraulic strain energy accumulator by using elastomer with strain energy storage mechanism to obtain higher energy storage efficiency and energy density. Based on this, John T. Tucker [9] evaluated and verified the materials, and found that the energy density of polyurethane was $15.4 \mathrm{~kJ} / \mathrm{kg}$ when the loaded stress was $17.23 \mathrm{MPa}$, the energy loss was $17.8 \%$, and then designed a low-pressure prototype (the maximum filled liquid pressure was $0.38 \mathrm{MPa}$ ), results showed that the energy storage efficiency of polyurethane in the accumulator exceeded $88 \%$. Then a high-pressure prototype (the maximum filled liquid pressure was 2.41MPa) was gained and a higher energy density was obtained. Daniel N. Cramer and Eric J. Bart [10] conducted charging/discharging tests on rubber airbags of different materials, and concluded that latex airbag had ideal expansion and contraction behavior, and obtained the energy efficiency of the airbag with different inner diameters and materials. John M. Tucker and Eric J. Barth [11] established the mathematical model of energy density of hydraulic strain energy accumulator and analyzed the factors that affected energy density and gave corresponding solutions. Joshua J. Cummins et al. [12] established mathematical model of the charging/ discharging energy storage efficiency of pneumatic strain energy accumulators based on enthalpy analysis method, and explored the influence of the rubber Mullins effect on the expansion pressure, contraction pressure and energy storage efficiency of the device. But it did not reveal the energy loss inside the device.

From the above analysis, it is known that the expansion pressure of the strain energy accumulator based on rubber material is an effective energy storage device with high energy density and steady pressure output. The pressure is determined by material, wall thickness and radial expansion radius. However, there are two more problems needed to be further focused that the above researches have not involved. On the one hand, in order to adapt to system different pressure, it is necessary to customize the airbag. On the other hand, the device performance depends heavily on rubber material characteristics. With the increase of use times, the rubber material damage will increase, which will inevitably lead to the decline of the performance of the whole device.

The key to solve the two problems is to do accurate energy analysis, which should be carried out simultaneously from the two perspectives of energy quantity and quality. The common enthalpy energy analysis method only analyzes the energy storage efficiency from the perspective of the amount of energy, and cannot reveal the qualitative changes of energy during the conversion or transfer process. At the same time, the method cannot fully reflect the loss within the system [13]. At present, an energy-saving analysis method widely used in industry is the exergy analysis method, which combines the "quantity" and "quality" of energy, scientifically analyzes the utilization of energy conversion and transmission, and it can truly reflect the external and internal losses in the process of energy utilization [14]. Kamran T and Rainer G [15] analyzed the energy-saving optimization scheme of industrial compressed air system based on the combination of exergy analysis method and energy efficiency method. The results showed that the exergy analysis method was more in line with the actual situation and could make more accurate decisions on energy saving of compressed air system. Hamidreza M and Xiaolin Wang [16] used exergy analysis to study the performance of the main components of a single-stage adiabatic compressed air energy storage system under different working conditions.

To sum up, based on the work of literature [12], this paper mainly focuses on accurate energy storage efficiency model and influence of Mullins effect during the process of rubber airbag charging/discharging by 
exergy analysis. First, the structure of the constant pressure elastic strain energy accumulator and the principle of constant pressure is explained. Then the specific modeling process is given, and a constant pressure elastic strain energy accumulator charging/discharging test platform is built to verify the difference between exergy analysis method and the enthalpy analysis method. Finally, combining with uncertainty analysis, the influence of Mullins effect on the charging/discharging performance of the rubber airbag is explored.

\section{Elastic strain energy accumulator design}

The structure of the constant pressure strain energy accumulator is shown in Fig 1, which is mainly composed of rubber airbag, rigid shield, fixed ring, quick coupling, etc. The rubber airbag is to store energy, the principle is that when the airbag is charged into the gas, the compressed gas does work on the airbag so the airbag is expanded, and the gas pressure energy converts into strain energy and is stored in the elastic body. When the airbag contracts and does work on the gas, the elastic body strain energy converts into pressure energy and releases the stored energy. The rigid shield is to limit the rubber airbag radial strain, on the one hand, it can increase the pressure during the charging/discharging process; on the other hand, it can prevent the rubber airbag from reaching the ultimate strain every expanded time and causing premature fatigue to ensure the device performance. The fixing ring is connected with the rigid shield and the quick coupling by interference fit to limit the position of the rigid shield and the quick coupling.

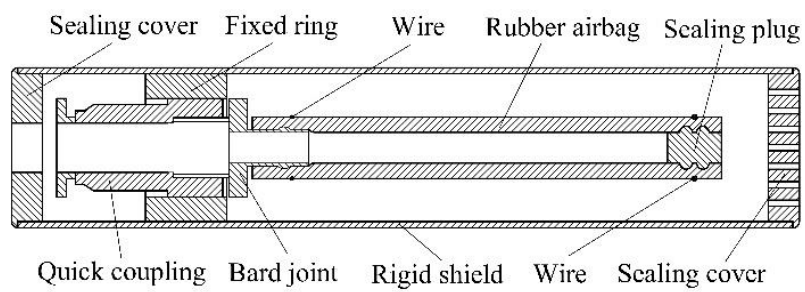

Figure 1 Constant pressure elastic strain energy structure

The constant pressure elastic strain energy accumulator characteristic is that it can expand and contract at a relatively constant pressure when charging/discharging. The charging/discharging pressure-volume curve is shown in Fig 2, and the rubber material typical stress-strain curve is shown in Fig 3.

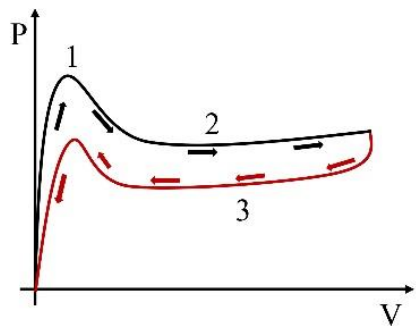

Figure 2 Charging/discharging P-V curve of rubber airbag

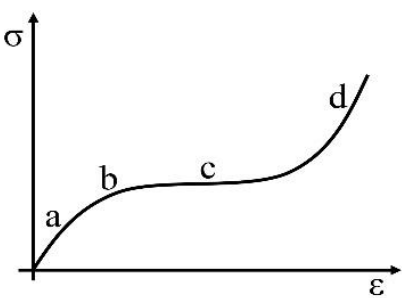

Figure 3 Stress-strain curve of hyperelastic rubber

The stress-strain curve in Fig 3 was divided into four regions. Region_(a) is the initial elastic modulus of rubber, which corresponds to the gas pressure rise stage in Fig 2 . As more gas is filled, the stress on the rubber airbag increases, and the elastic modulus begins to decrease, corresponding to area_(b) in Fig 3. At this time, the rubber airbag undergoes hyperelastic deformation and begins to expand, resulted in a sudden increasement in the airbag volume. The gas pressure decreases as shown in Fig 2 . Subsequently, the pressure continues to decrease until the elastic modulus of the expansion zone of the rubber airbag increases as shown in the region_(d), the elastic modulus at this stage is the same as the region_(a). The local increase of the elastic modulus is caused by the local strain hardening of the rubber material. At this time, the expansion area of the rubber airbag begins to move along the axial direction of the airbag with the minimum elastic modulus in the region_(c). When the expansion area moves in the axial direction, the pressure is not changed, while the volume continues to increase, corresponding to the flat area in Fig 2.

The energy loss during the charging/discharging process is mainly caused by three factors: (1)the rubber material mechanical properties(such as the Mullins effect, hysteresis effect, etc. causing material loss); (2)the friction between the airbag and rigid shield during the charging/discharging process causes the energy loss; (3) Pressure loss due to the thermal change. The impact of the three factors on the rubber airbag directly reflected in the value of its contraction pressure lower than the expansion pressure, which leads to energy loss. The main factor 
considered when analyzing the energy storage efficiency of the rubber airbag was the relationship between the expansion pressure and the contraction pressure of the rubber airbag.

\section{Exergy storage efficiency analysis}

\subsection{Enthalpy analysis method}

The calculation of the strain energy generated by the charging/discharging of the airbag by the enthalpy analysis method was based on the idea of the expansion energy to do work as shown in Fig 4.

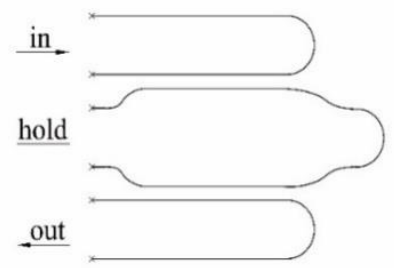

Fig 4 Charging/discharging process of rubber airbag: Top: charged; Middle: hold; Bottom: discharged

During charging, the gas expands and works to airbag, the airbag volume is charged. The gas pressure energy converts into elastic material strain energy and is stored in the elastic body. The energy was calculated as shown in Formula (1).

$$
E_{\text {Tot }}=E_{s}+E_{p}=\int_{V_{0}}^{V_{\text {full }}} P_{\exp } d v+P_{\text {exp }} V_{\text {full }} \ln \left(\frac{P_{\text {exp }}}{P_{\text {atm }}}\right)
$$

where $E_{\text {tot }}$ is the total energy of entered the airbag, $E_{s}$ is the strain energy produced by the expansion of the rubber material, $E_{P}$ is the gas pressure energy stored inside the airbag, $V_{0}$ is the initial volume of the airbag, $V_{\text {full }}$ is the volume of the airbag after inflation, $P_{\exp }$ is the inflation pressure, and $P_{\text {atm }}$ is the atmospheric pressure.

The discharging process is the inverse process of the charging process, and only need to modify corresponding integral limit. The ratio of discharging energy to charging energy is the energy storage efficiency of the airbag during charging/discharging process as shown in formula (2).

$$
\eta=\frac{-\int_{V_{\text {full }}}^{V_{0}} P_{c o n} d v+P_{c o n} V_{\text {full }} \ln \left(\frac{P_{c o n}}{P_{\text {atm }}}\right)}{\int_{V_{0}}^{V_{\text {full }}} P_{\text {exp }} d v+P_{\text {exp }} V_{\text {full }} \ln \left(\frac{P_{\text {exp }}}{P_{a t m}}\right)}
$$

Where $P_{c o n}$ is Contraction pressure.

It can be seen from formula (2) that when calculating the charging/discharging energy storage efficiency, it is necessary to know the expansion pressure, contraction pressure and expansion volume. The airbag volume is a dynamic change process, and the expansion volume and contraction volume at any time cannot be obtained by measurement, so the ideal gas state equation was used to calculate the airbag volume at any time during the charging/discharging process, which will cause a certain error. At the same time, the enthalpy analysis method cannot reflect the thermal changes influence on the energy storage efficiency, so it is necessary to analyze the energy storage efficiency of rubber airbags based on the exergy analysis method.

\subsection{Exergy analysis method}

Since energy conversion is related to environmental conditions and process characteristics, in order to measure the maximum energy conversion capacity, it is necessary to determine the zero energy state when calculating the exergy balance. This article adopts the environmental standards specified in GB/T 14909-94, $P_{0}=0.1 \mathrm{MPa}, T_{0}=298.15 \mathrm{~K}$.

The energy storage efficiency exergy analysis of the rubber airbag was based on the open thermal system exergy equation, and the following assumptions were made: (1)The charging/discharging process is adiabatic, there is no heat exchange with the external environment; (2) The gas is assumed to be ideal gas, the kinetic and potential energy exergy of the gas are ignored; (3)During the discharging phase, the gas can be completely discharged from the airbag; (4) The entire charging/discharging process is a steady state and steady flow condition.

Based on the above assumptions, the system exergy change during charging can be obtained as formula (3).

$$
e_{x .1}-e_{x .2}=w_{s}+l
$$

where $w_{s}$ is the output work in the charging stage, that is, the work done by the gas is charged into the airbag, that is, the strain energy $e_{s}$ generated by the airbag inflation converted by the pressure energy. $e_{x .1}$ and $e_{x .2}$ are the specific flow exergy before and after the gas enters the airbag, which are calculated as formulas (4) and (5), $l$ is the exergy loss, which mainly includes the damage of the rubber material during the conversion of pressure energy to strain energy. 


$$
\begin{aligned}
& e_{x .1}=c_{p}\left(T_{1}-T_{0}\right)-T_{0}\left(c_{p} \ln \frac{T_{1}}{T_{0}}-R_{g} \ln \frac{p_{1}}{p_{0}}\right) \\
& e_{x .2}=c_{p}\left(T_{2}-T_{0}\right)-T_{0}\left(c_{p} \ln \frac{T_{2}}{T_{0}}-R_{g} \ln \frac{p_{2}}{p_{0}}\right)
\end{aligned}
$$

Where $P_{0}$ and $T_{0}$ are the pressure and temperature of the reference environment, $P_{1}$ and $T_{l}$ are the pressure and temperature before the gas enters the airbag, $P_{2}$ and $T_{2}$ are the pressure and temperature after the gas enters the airbag to perform work on the airbag, $R g=0.287 \mathrm{~kJ} /(\mathrm{kg} \cdot \mathrm{K})$ is the gas constant of air, and $c_{p}=1.004 \mathrm{~kJ} /(\mathrm{kg} \cdot \mathrm{K})$ is the constant pressure specific heat of air.

In the first stage, the gas is charged the accumulator to apply work on the airbag. Assuming that the specific flow of the gas entering the airbag through the inlet of the control surface at this stage is $e_{x .1}$; the gas is charged the airbag and works on the airbag, and the strain energy generated after the airbag is stably expanded is $e_{s . l}$, define $e_{s .1}$ as the revenue exergy of the system, $e_{x .1}$ as the expenditure exergy, and the ratio of the two is defined as the exergy efficiency of the inflation stage as shown in formula (6).

$$
\eta_{e x .1}=\frac{e_{s .1}}{e_{x .1}}
$$

In the second stage, the gas is discharged, and the airbag worked on the gas to convert strain energy into pressure the airbag through the outlet of the control surface at this stage is $e_{x .2}$, and $\mathrm{e}_{s .1}$ is defined as the payment exergy of the system, $e_{x .2}$ is the revenue exergy of the system, and the ratio of the two is defined as the exergy efficiency in the discharging stage, as shown in formula (7).

$$
\eta_{e x .2}=\frac{e_{x .2}}{e_{s .1}}
$$

Regarding the two stages as a whole, that is, the gas works on the airbag after charging to expand the airbag, and the airbag contracts when discharging. The exergy efficiency of the entire system is showed in formula (8).

$$
\eta_{e x .3}=\eta_{e x .1} \cdot \eta_{e x .2}=\frac{e_{s .1}}{e_{x .1}} \cdot \frac{e_{x .2}}{e_{s .1}}=\frac{e_{x .2}}{e_{x .1}}
$$

rubber airbag, this paper mainly verified the following problems: (1) The steady-state value of the expansion pressure, contraction pressure and energy storage efficiency during the cyclic charging/discharging process; (2)The exergy analysis method and the enthalpy analysis method were compared to verify the advantages of the exergy analysis method in calculating the rubber airbags energy storage efficiency.

\subsection{Experimental device and principle}

The test platform is shown in Fig 5, composed of a two-way mass flow sensor, a throttle valve, a pressure sensor, a temperature sensor, a three-position five-way solenoid valve, a power supply, a data acquisition card and control procedure (the acquisition control terminal was not shown in the figure). The constant pressure elastic strain energy accumulator used in the test was composed of a rubber air bag with an inner diameter of $5 \mathrm{~mm}$ and an outer diameter of $10 \mathrm{~mm}$ and a rigid protective cover with an inner diameter of $29 \mathrm{~mm}$.

The air supply pressure was adjusted to $0.4 \mathrm{MPa}$ by the pressure regulator. The compressed gas flowed through the three-position five-way solenoid valve through the bidirectional mass flowmeter, pressure sensor and temperature sensor, and then entered the rubber airbag. The three-way solenoid valve became a three-way valve by blocking an intake hole, and the charging/discharging cycle through three processes: First, in the charging stage, the solenoid valve spool moved to the left to connect the solenoid valve air inlet; The second stage was the holding stage, the solenoid valve spool moved to the middle position, the air inlet was closed, and the airbag kept expanding; Finally, in the discharging stage, the solenoid valve spool moved to the right. Because the air inlet was blocked, the gas would not enter the air bag, but the gas in the air bag was discharged into the atmosphere through the left exhaust port of the solenoid valve. Bidirectional mass flowmeter, pressure sensor and temperature sensor were used to measure the mass flow, pressure and temperature in and out of the airbag. Two throttle valves were installed on both sides of the mass flowmeter to control the gas flow within the measurement range of the mass flowmeter. The opening and closing of solenoid valve and data acquisition were programmed in the Simulink.

\section{Experimental verification}

Through the cyclic charging/discharging tests of the 


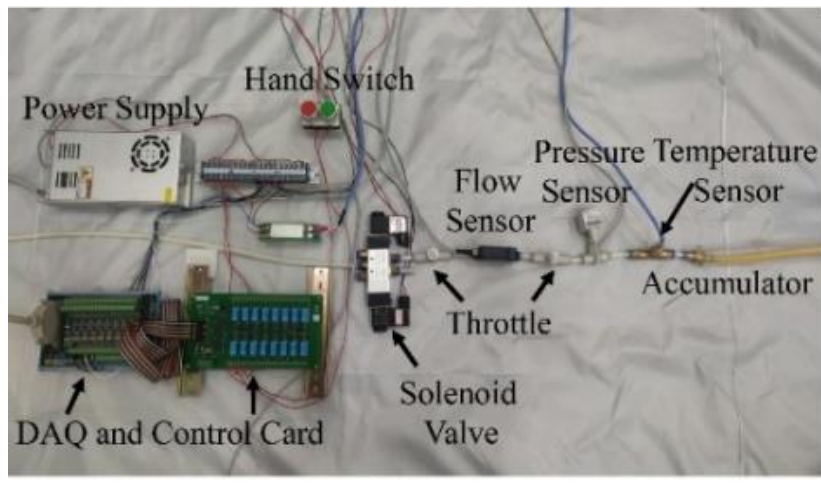

Fig 5 Constant pressure strain energy accumulator charging /discharging energy storage efficiency test bench

A charging/discharging cycle took $5 \mathrm{~s}$, including charging time $0.8 \mathrm{~s}$, holding for $2 \mathrm{~s}$, and then discharging. Five charging/discharging periods were set for the tested airbag, and each period includes 40 charging/discharging cycles, and a 10 minute recovery period was set between each period. The data of five periods were processed as independent samples. In order to weaken the Mullins effect, 15 pre-inflation cycles were performed before the test. All pressures were converted to absolute pressures.

The airbag volume dynamic change in charging stage is shown in Figure 6. At the initial charging stage, the airbag formed a bubble, with the continuous gas was filled, the bubble continued to expand radially until it contacted with the rigid shield, and then continued to expand along the axial direction.

It should be noted that the bubble location is not fixed. When the bubble appears at the top (away from the charging end) or the middle of the airbag, it will lead to the rubber airbag undesirable expansion behavior as shown in Figure 7. In this way, the rubber airbag cannot expand completely, and the energy storage capacity will be greatly reduced. Daniel N Cramer [10] observed the same phenomenon when experimenting with different rubber airbags. In order to obtain the ideal expansion behavior, the airbag basement (gas inlet) was pre-strained before charging to make the bubble appear at the airbag basement. At the same time, medical lubricant was used to lubricate the airbag and the rigid shield to reduce the friction.

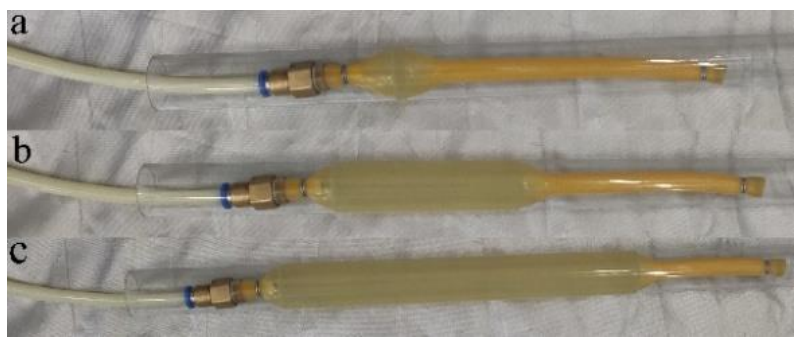

Fig 6 Airbag charging process: a) bubbles appear; b) bubble expand along the axial direction; c) expansion completed

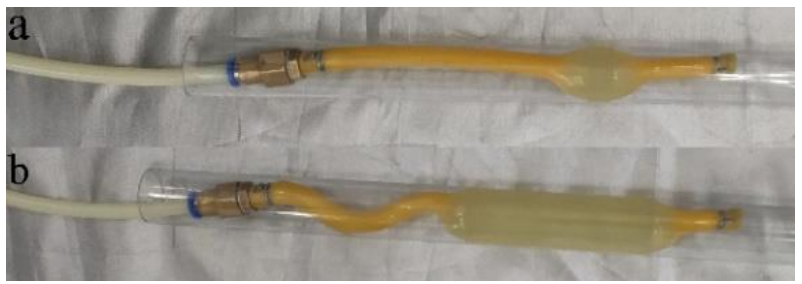

Fig 7 Non-ideal expansion behavior of airbag

Obtaining the pressure, flow and temperature of the airbag from the sensor. The value of a certain cycle is shown in Figure 8 -10.

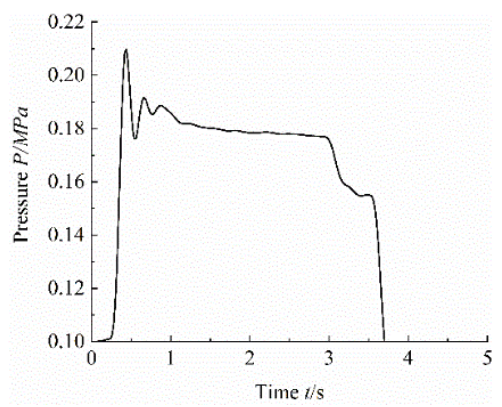

Fig 8 Relationship between pressure and time of a charging/discharging cycle

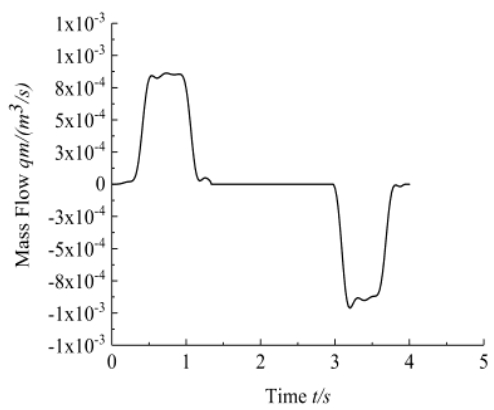

Fig 9 Relationship between mass flow and time of a charging/discharging cycle 


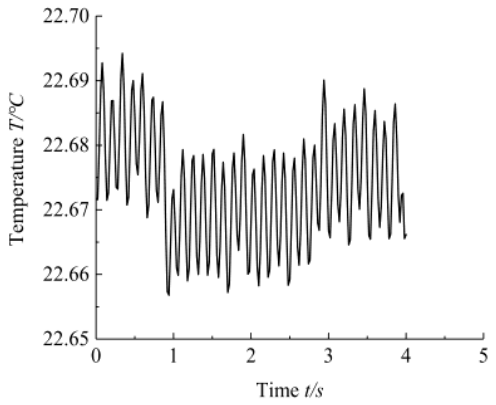

Fig 10 The relationship between temperature and time of a charging/discharging cycle

For Fig 8, it can be seen that in initial charging stage, gas continued to enter the airbag, and the pressure continued to rise, which will form a pressure peak until the bubble was formed. When the bubble formed, the pressure began to drop. A slight decrease in pressure during the maintenance phase indicated existed gas leakage. The airbag experienced a relatively constant pressure contraction when discharging. The positive peak of the mass flow curve in Fig 9 represented the charging phase, and the negative peak represented the discharging phase. The temperature change of a charging/discharging cycle is shown in Fig. 10. The difference between the maximum temperature and the minimum temperature was only $0.04{ }^{\circ} \mathrm{C}$, indicating that the charging/discharging process is an isothermal process.

\subsection{Data processing and calculation}

It is necessary to know the expansion pressure, contraction pressure, gas temperature and gas quality of each cycle when calculating the rubber airbag energy storage efficiency.

The gas quality entering and leaving the airbag can be obtained by integrating the mass flow. The integration of the mass flow will cause data drift [12]. The integrated gas quality was adjusted according to the three adjustment principles proposed in the literature [12]:(1)The total mass of each cycle should start and end from zero; (2)The later data points have a larger uncertainty range than the previous data points; (3) The overall shape of the data can be scaled, but should remain unchanged. The quality and adjusted data of the gas entering and exiting the airbag during a charging/discharging cycle are shown in Fig 11.

After integrating the mass flow rate, the airbag volume change at any time can be obtained by the ideal gas state equation, and then the pressure-volume curve of charging/discharging cycle can be obtained by combining the volume and pressure at each time, as shown in Figure 12. The red solid line represents the charging stage, and the blue dotted line represents the discharging stage. It can be seen that the airbag volume experienced continuous expansion/contraction and relatively constant pressure in the charging/discharging stage, indicating the rubber airbag had constant pressure charging/discharging characteristics.

The pressure average value between two squares represents expansion pressure and pressure average value between two triangles represents contraction pressure. The expansion pressure and contraction pressure of the airbag in 5 test cycles are shown in Fig 13.

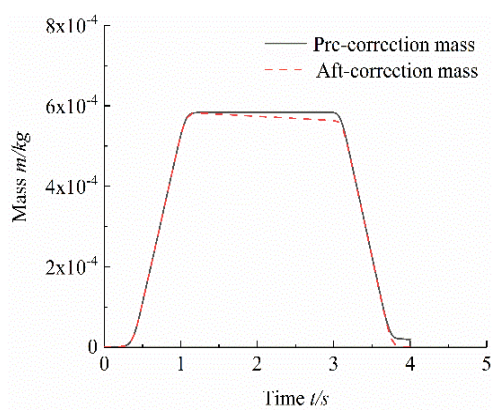

Fig 11 Relationship between gas quality and time before and after adjustment

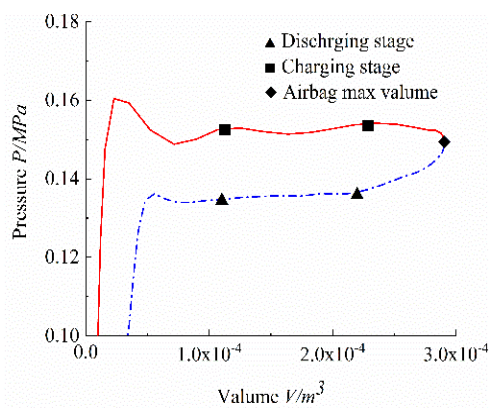

Fig 12 Relationship between the pressure and volume of a charging/discharging cycle

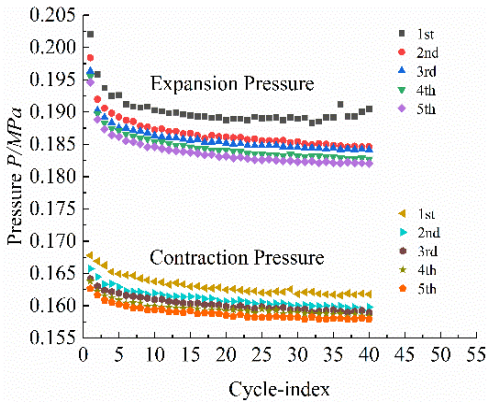

Fig 135 test periods changes in expansion pressure and contraction pressure

From Figure 13, it can be seen that the expansion pressure and contraction pressure decreased in five test 
periods, which indicated the Mullins effect influences on expansion pressure and contraction pressure. The expansion pressure and contraction pressure in the initial charging/discharging stage of the latter test cycle were both greater than those in the latter half of the previous cycle, which was caused by the fact that the airbag experienced a 10-minute recovery period after each test period. In the second half of each period, the expansion pressure and contraction pressure decreased slowly, which indicate that the rubber material has good resilience.

Expansion pressure, contraction pressure and temperature data obtained from each charging/discharging cycle were substituted into Formula (6) and (7) to obtain the exergy changes of the gas inside and outside the airbag, as shown in Fig 14. Exergy storage efficiency of airbag charging/discharging cycle can be calculated from Formula (8), as shown in Fig 15.

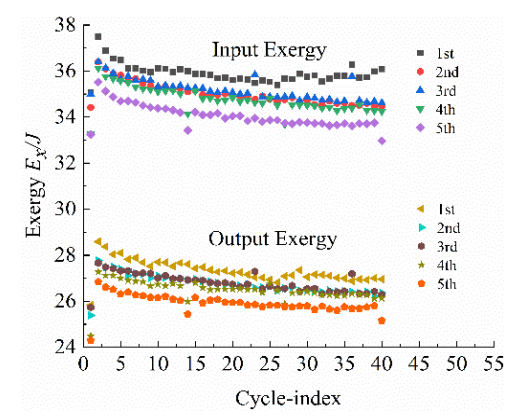

Fig 145 test periods in and out of the airbag exergy value

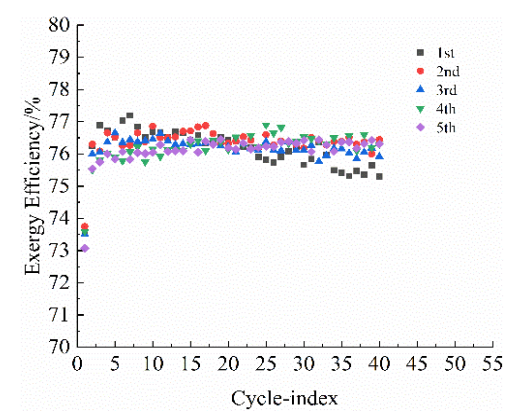

Fig 15 Efficiency changes in 5 charging/discharging periods

According to Fig 14, it can be seen that the energy storage efficiency in the first cycle was lower, improved and tended to be stable in the subsequent cycles, because the rubber airbag was not softened under the loaded before the first charging/discharging cycle, so in the first cycle, the pressure energy had a large loss during transforming into strain energy. In the subsequent cycles, because the rubber airbag had been softened, the energy loss will be reduced when the gas overcame the elastic force of the rubber airbag and worked to convert the pressure energy into strain energy. The energy storage efficiency will be significantly improved and stabilize in the subsequent cycles.

\section{Steady state characteristic analysis}

The main indexes to measure the constant pressure elastic strain energy accumulator quality are whether the expansion pressure, contraction pressure and energy storage efficiency can always keep stable in charging/discharging cycles. As an energy storage component, due to the Mullins effect and hysteresis effect, the rubber airbag performance inevitably decreased during the charging/discharging cycles. The performance changes eflected in the changes of expansion pressure, contraction pressure and energy storage efficiency. Through the uncertainty analysis to determine whether the airbag performance after multiple charging/discharging cycles can meet the use requirements.

Calculated the average $\mu$, standard deviation $\sigma$ and the standard deviation $S D_{\mu}$ of the average value of 40 cycles of each test as shown in formula (9)(10)(11).

$$
\mu=\frac{1}{n} \sum_{n=1}^{n} x_{i}
$$

$$
\begin{gathered}
\sigma=\left[\frac{1}{n-1} \sum_{i=1}^{n}\left(x_{i}-\mu_{x}\right)^{2}\right]^{1 / 2} \\
S D \mu=\sigma / \sqrt{n}
\end{gathered}
$$

Where $n$ is the total number of data points, $x_{i}$ is the value of the $i$-th data point. Due to the Mullins effect, the data obtained from the first charging/discharging cycle will be very different from the subsequent data. Therefore, the first charging/discharging cycle data in every period was discarded in the uncertainty analysis. The average value and standard deviation of each parameter in the charging/discharging cycle are shown in Table 1 to 3 .

From table 1 and 2, it can be seen that the expansion pressure average value decreased by $6 \mathrm{e}-3 \mathrm{MPa}$ and the contraction pressure average value decreased by $4 \mathrm{e}-3 \mathrm{MPa}$ in the five test periods, and the standard deviations were $2.92 \mathrm{e}-3 \mathrm{mpa}$ and $1.79 \mathrm{e}-3 \mathrm{mpa}$ respectively. According to the table 3 , it can be seen that the energy storage efficiency average value change in five test periods was $0.2 \%$, and the standard deviation was $0.317 \%$. The above 
data show that the rubber airbag has good mechanical properties in multiple charging/discharging cycles.

Table 1 Expansion pressure Average value and standard deviation

\begin{tabular}{ccc}
\hline Test & Mean(MPa) & Standard deviation(MPa) \\
\hline 1 & 0.190 & 0.00243 \\
2 & 0.186 & 0.00175 \\
3 & 0.186 & 0.00146 \\
4 & 0.184 & 0.00168 \\
5 & 0.184 & 0.00162 \\
5 cycle average & 0.186 & 0.00292 \\
\hline
\end{tabular}

Table 2 Contraction pressure average value and standard deviation

\begin{tabular}{ccc}
\hline Test & Mean(MPa) & Standard deviation(MPa) \\
\hline 1 & 0.163 & 0.00131 \\
2 & 0.161 & 0.00118 \\
3 & 0.160 & 0.00105 \\
4 & 0.159 & 0.00087 \\
5 & 0.159 & 0.00090 \\
5 cycle average & 0.160 & 0.00179 \\
\hline
\end{tabular}

Table 3 Energy storage efficiency average value and standard deviation

\begin{tabular}{ccc}
\hline Test & Mean $(\%)$ & Standard deviation $(\%)$ \\
\hline 1 & 76.2 & 0.510 \\
2 & 76.4 & 0.202 \\
3 & 76.2 & 0.197 \\
4 & 76.3 & 0.294 \\
5 & 76.2 & 0.209 \\
5 cycle average & 76.3 & 0.317 \\
\hline
\end{tabular}

In the previous work, the energy storage efficiency model was established based on the enthalpy analysis method and the experimental analysis was carried out (the results are shown in Table 4). In 200 charging/discharging cycles, the energy storage efficiency error was $0.584 \%$, and the energy storage efficiency was $76.9 \%$.

Table 4 Energy storage efficiency average and standard deviation

\begin{tabular}{ccc}
\hline Test & Mean $(\%)$ & Standard deviation $(\%)$ \\
\hline 1 & 76.4 & 0.499 \\
2 & 76.8 & 0.559 \\
3 & 76.8 & 0.524 \\
4 & 76.6 & 0.583 \\
5 & 76.5 & 0.541 \\
5 cycle average & 76.9 & 0.584 \\
\hline
\end{tabular}

It can be seen from table 3 and 4 that the standard deviation of the energy storage efficiency average value calculated based on the enthalpy analysis method was $0.317 \%$, and the standard deviation of the energy storage efficiency average value calculated based on the enthalpy analysis method was $0.584 \%$, which indicates that the energy storage efficiency accuracy calculated by the exergy analysis method is higher than that calculated by the enthalpy analysis method.

\section{Conclusion and outlook}

Aiming at the problem of insufficient energy storage efficiency modeling with enthalpy analysis method, this paper proposed to establish the rubber airbag charging/discharging energy storage efficiency model based on exergy analysis method and built the test platform to verify energy storage efficiency. The following conclusions were drawn: (1) The experiment confirmed that the energy storage efficiency model error based on the exergy analysis method reduced by $0.267 \%$ compared with the enthalpy analysis method, which proved the exergy analysis method relative accuracy; (2) The Mullins effect mainly reflected in the expansion pressure and contraction pressure changes during charging/discharging cycles. The expansion pressure average value decreased by $6 \mathrm{e}-3 \mathrm{MPa}$ and contraction pressure average value decreased by $4 \mathrm{e}-3 \mathrm{MPa}$, and the standard deviation were $2.92 \mathrm{e}-3 \mathrm{MPa}$ and $1.79 \mathrm{e}-3 \mathrm{MPa}$, respectively.

This paper verified that the constant pressure strain energy accumulator can store and release energy with relatively constant pressure, and had high energy storage efficiency. The future work plan is to explore the combination relationship between the size of rigid shield and rubber airbag to maximize the energy storage efficiency.

\section{Declaration}

\section{Acknowledgements}

The authors sincerely thanks to reviewers of the journal for critical discussion and reading during manuscript preparation.

\section{Funding}

Supported by the National Natural Science Foundation of China (51175053) and the Fundamental Research Funds for the Central Universities of China (3132020123 and 3132019352).

\section{Availability of data and materials}

The datasets supporting the conclusions of this article are included within the article. 


\section{Authors' contributions}

The author' contributions are as follows: Hong-Wang $\mathrm{Du}$ was responsible for the collection and processing of experimental data, Dong-dong Yang wrote the manuscript, Wei Xiong reviewed and revised the manuscript.

\section{Competing interests}

The authors declare no competing financial interests.

\section{Consent for publication}

Not applicable

\section{Ethics approval and consent to participate}

Not applicable

\section{References}

[1] Qiuhong Meng, Jingbo Guo. Application of regenerative braking technology in automobile [J]. Tianjin automobile, 2007 (03): 19-21 +40 .

[2] P Miller, C Cooper, F Kamakate, T Reinhart, M Kromer. Reducing heavy-duty long haul combination truck fuel consumption and $\mathrm{CO} 2$ emissions, NESCCAF-northeast states center for a clean air future, ICCT-international council on clean transportation, SWRI-southwest research institute, TIAX, LLC, final report October, 2009.

[3] N Hill, S Finnegan, J Norris, C Brannigan, D Wynn, H Baker, et al. Reduction and testing of greenhouse gas emissions from heavy duty vehicles-Lot 1: Strategy. Brussels: European Commission/AEA; 2 2011.

[4] Yaqian Liu. Research on energy storage density and efficiency of potential energy storage device [D]. Taiyuan University of science and technology, 2017.

[5] Yunsong Lu. Research on automotive accumulator [J]. Hydraulic and pneumatic, 2007 (07): 7-9.

[6] Qingyong Zhang. Research on accumulator characteristics of hydraulic hybrid electric vehicle [J]. Mechanical science and technology, 2011, 30 (11): 1911-1916.

[7] Bo Wang, zhongyun Yu, Shujun Yang, Xianzhi Tang. Configuration analysis of hydraulic hybrid electric vehicle based on composite accumulator [J]. Mechanical design, 2018, 35 (10): 15-23.

[8] A Pedchenko, E J Barth. Design and validation of a high energy density elastic accumulator using polyurethan. Proceedings of the ASME Dynamic System and Control Conference, PP. 283-290.

[9] J Tucker. Design and experimental evaluation of a high energy density elastomeric strain energy accumulator Master's thesis. Vanderbilt University; 2012.

[10] D N Cramer, E J Barth. Pneumatic strain energy accumulators for exhaust gas recycling.2014. ASME/BATH 2013 Symposium on Fluid Power and Motion Control

[11] J M Tucker, E J Barth. "Design, Fabrication, and Evaluation of a Distributed Piston Strain-Energy Accumulator," International Journal of Fluid Power, Vol. 14, No. 1, pp. 47-56, March 2013.

[12] J J Cummins, E J Barth, D E Adams. 2015. Modeling of a pneumatic strain energy accumulator for variable system configurations with quantified projections of energy efficiency increases. In: Proceedings of the 2015 Bath/ASME symposium on fluid power and motion control, 14 October Chicago, IL. New York, NY: American Society of Mechanical Engineers, V001T01A055-8.

[13] H Isam. Aljundi. Energy and Exergy Analysis of a Steam Power Plant in Jordan [J]. Applied Ther-Mal Engineering, 2009, 29(2-3): 324-328.

[14] Yuanyuan Jiang, Shaoxiang Zhou. Study on Evaluation Method for Regenerative Feed Heating System of Power Plant[C], 2020 International Conference on Advances in Energy Engineering, June19-20, 2010, Beijing, C.

[15] K Taheri, R Gadow. Industrial compressed air system analysis: Exergy and thermo economic analysis. CIRP Journal of Manufacturing Science and Technology, v 18, p 10-17, August 2017.

[16] H Mozayeni, Xiaolin Wang, M Negnevitsky. Exergy analysis of a one-stage adiabatic compressed air energy storage system. Energy Procedia, v 160, p 260-267, 2019, 2nd International Conference on Energy and Power, ICEP2018.

[17] J J Cummins. Characterization of a Pneumatic Strain Energy Accumulator: Efficiency and First Principles Models with Uncertainty Analysis [D]. Nashville: Vanderbilt University, 2017.

\section{Biographical notes}

Hong-Wang Du, born in 1984, is currently a associate professor and MD candidate supervisor at Naval Architecture and Marine Engineering School, Dalian Maritime University, China. His main research interests include fluid power transmission, virtual assembly technology and marine salvage technology. Tel: +86-0411-84723725; E-mail: duhw_1984@dlmu.edu.cn

Dong-dong Yang, born in 1993, is currently a master candidate at Naval Architecture and Marine Engineering School, Dalian Maritime University, China.

E-mail: yd17516067775@163.com

Wei Xiong, born in 1972, is currently a professor and $\mathrm{PhD}$ candidate supervisor at Naval Architecture and Marine Engineering School, Dalian Maritime University, China. His main research interests include fluid power transmission, virtual assembly technology and marine salvage engineering equipment.

Tel: +86-0411- 84727060; E-mail: xiongwei@dlmu.edu.cn 
Figures

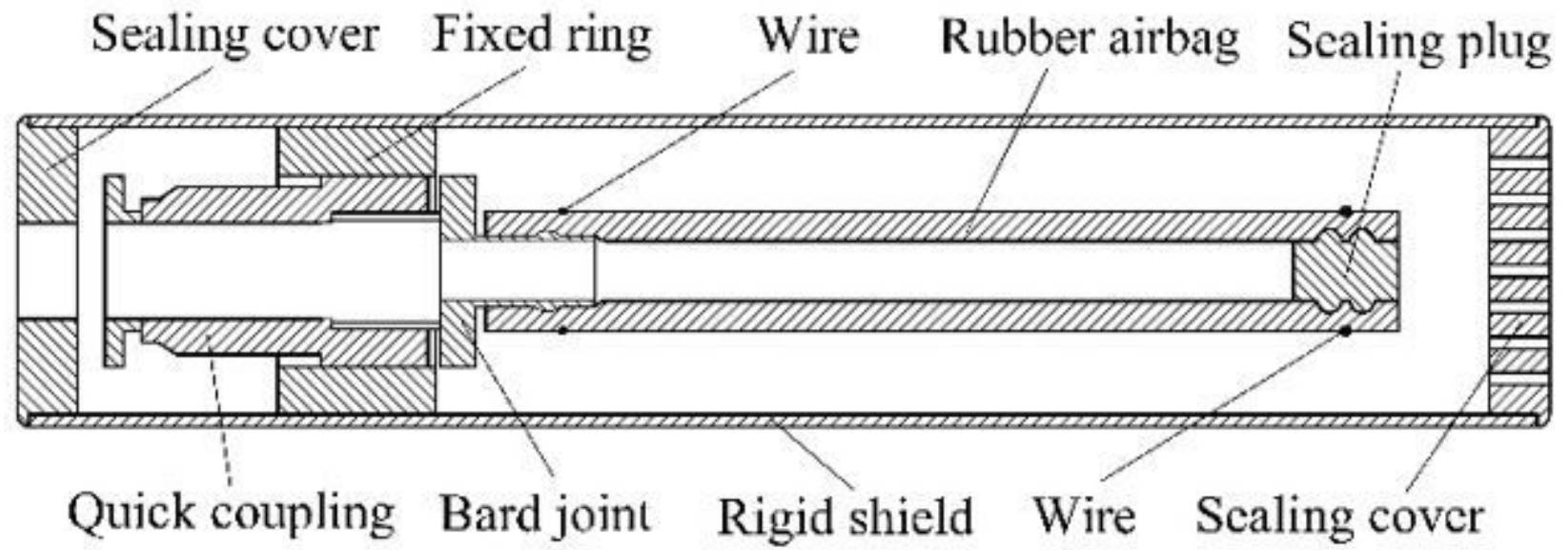

Figure 1

Constant pressure elastic strain energy structure

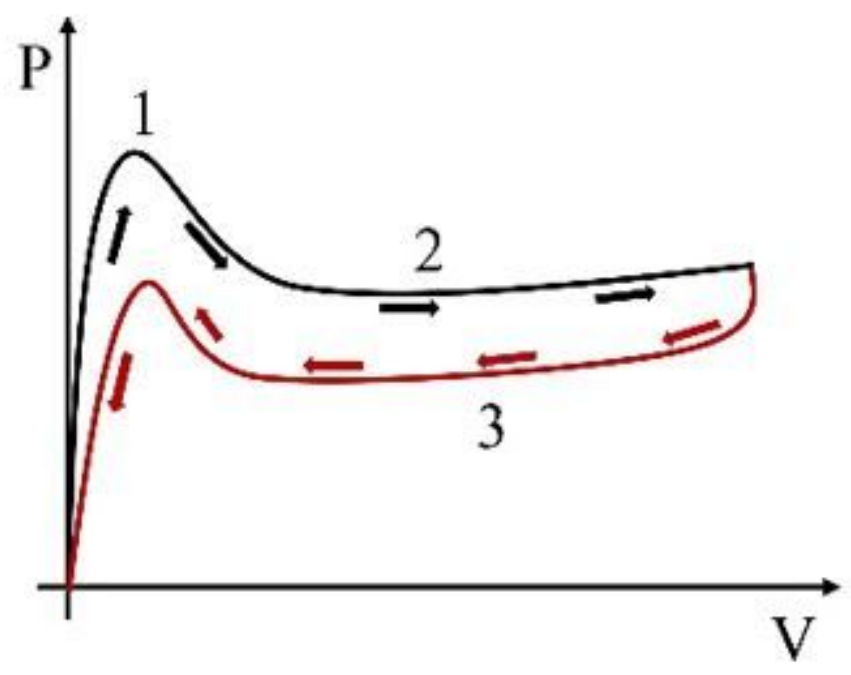

Figure 2

Charging/discharging P-V curve of rubber airbag 


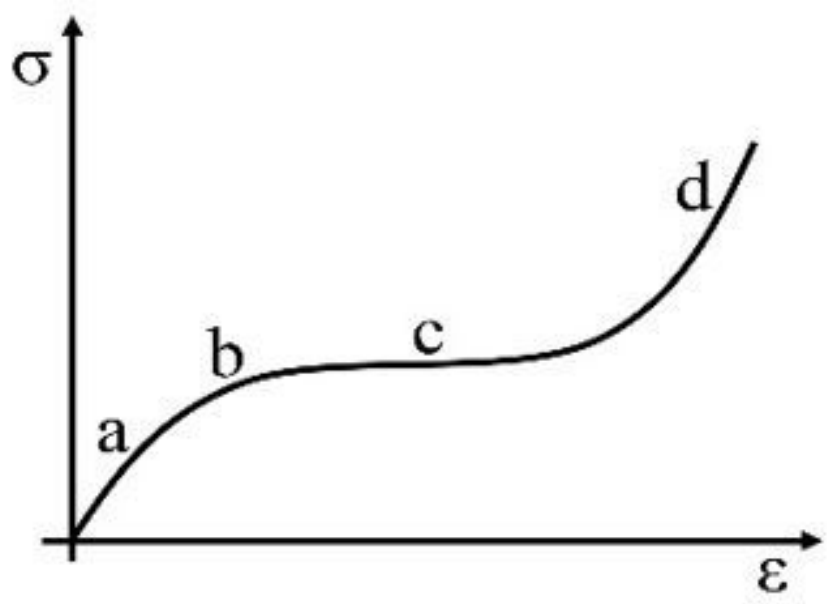

Figure 3

Stress-strain curve of hyperelastic rubber

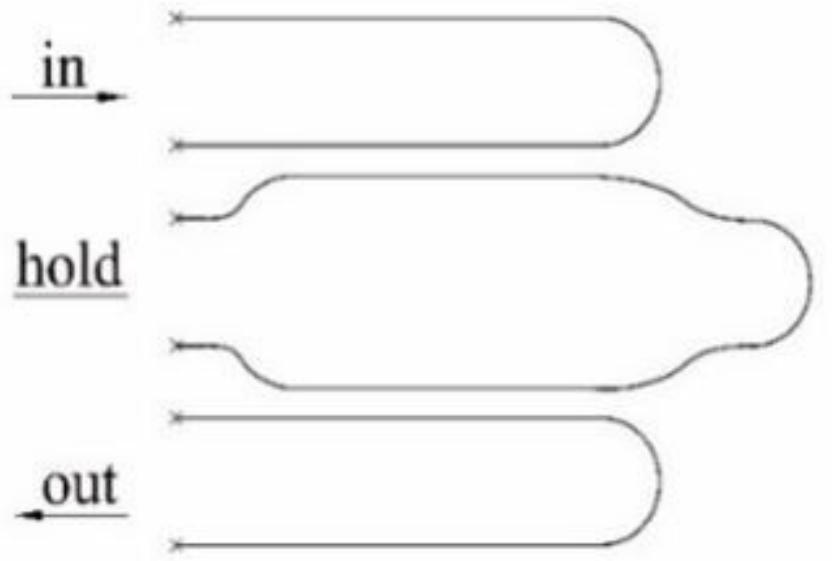

Figure 4

Charging/discharging process of rubber airbag: Top: charged; Middle: hold; Bottom: discharged 


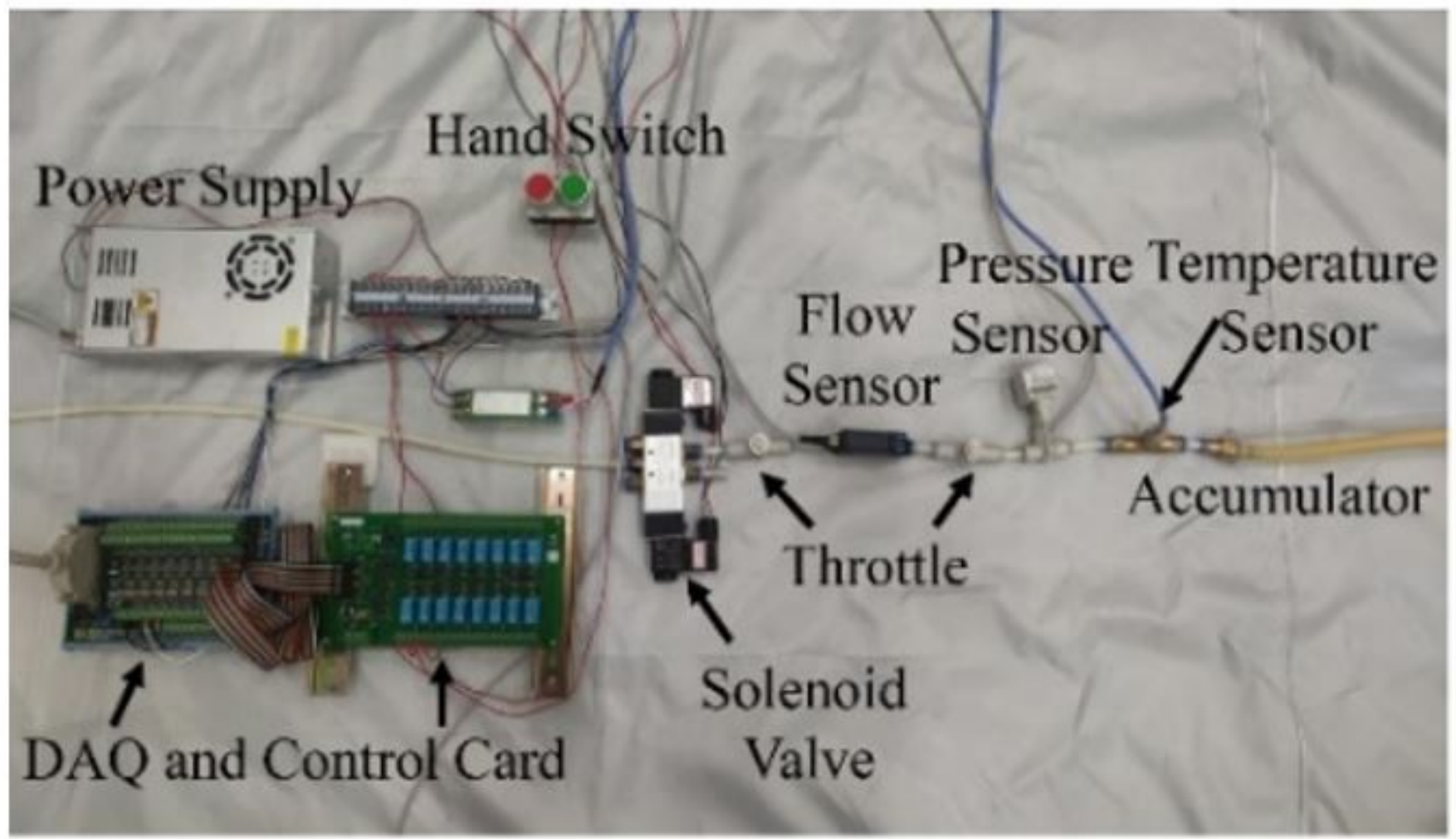

Figure 5

Constant pressure strain energy accumulator charging /discharging energy storage efficiency test bench

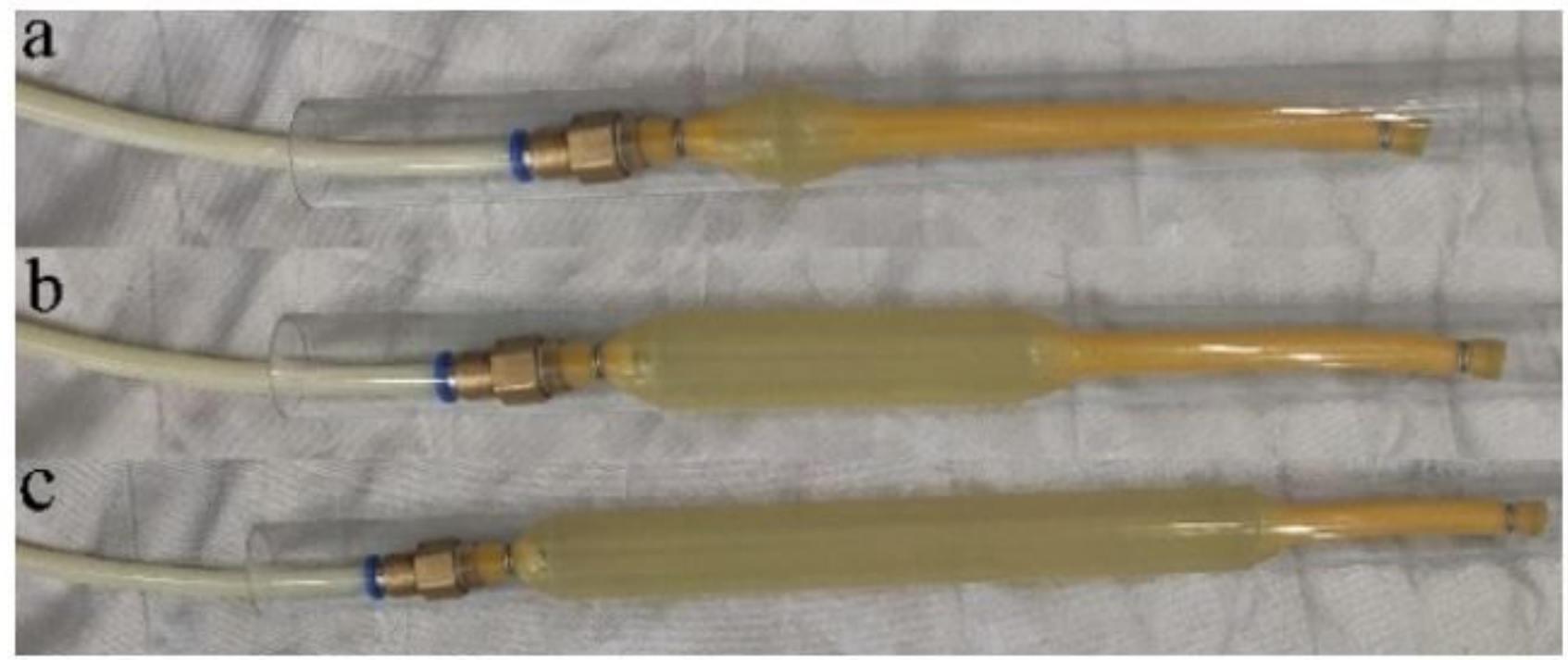

Figure 6

Airbag charging process: a) bubbles appear; b) bubble expand along the axial direction; c) expansion completed 


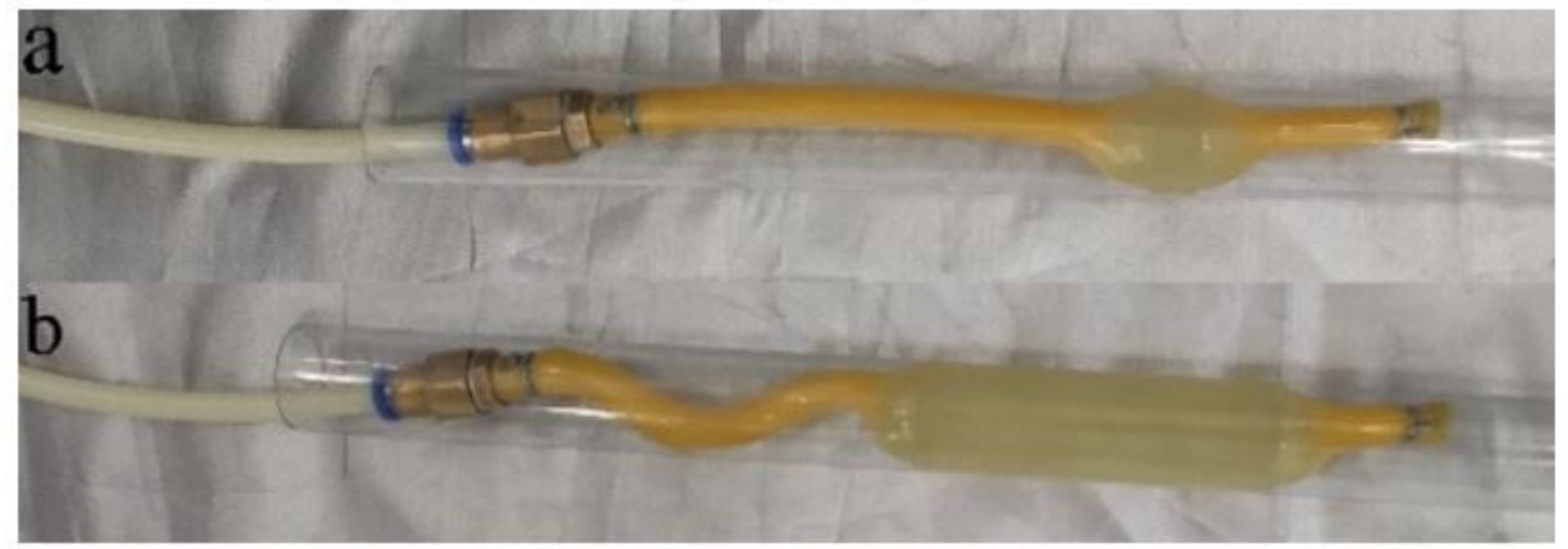

Figure 7

Non-ideal expansion behavior of airbag

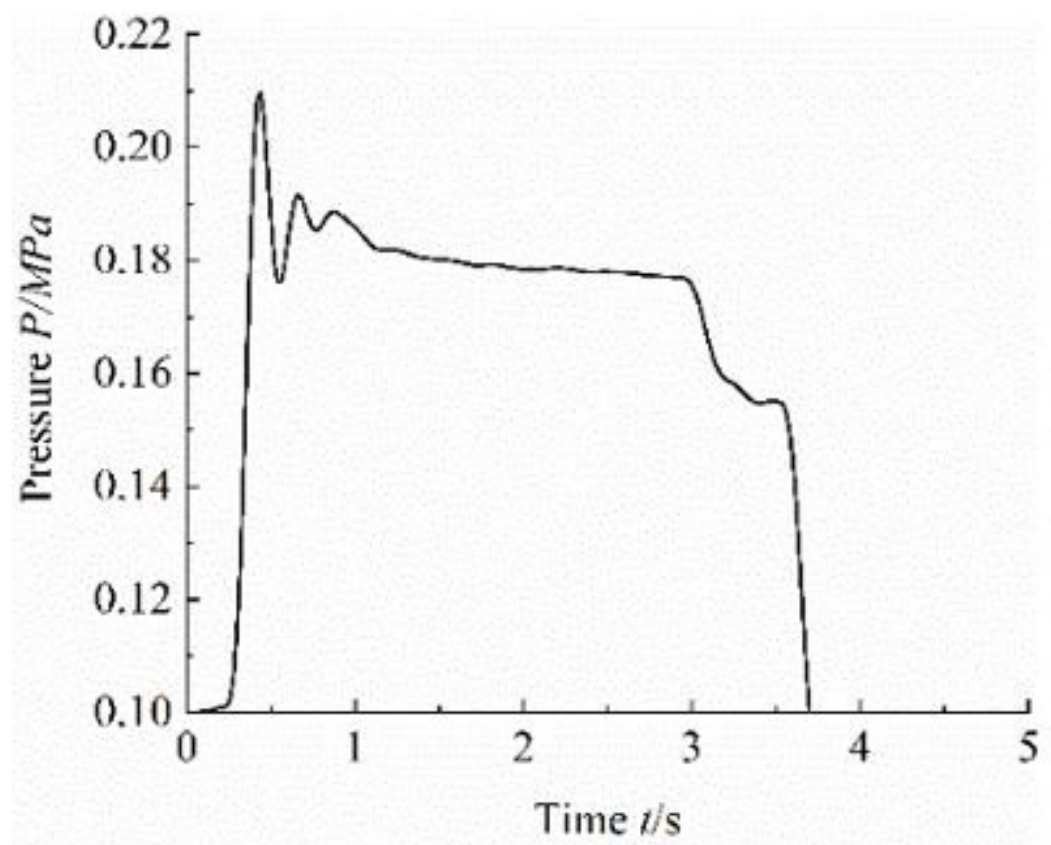

Figure 8

Relationship between pressure and time of a charging/discharging cycle 


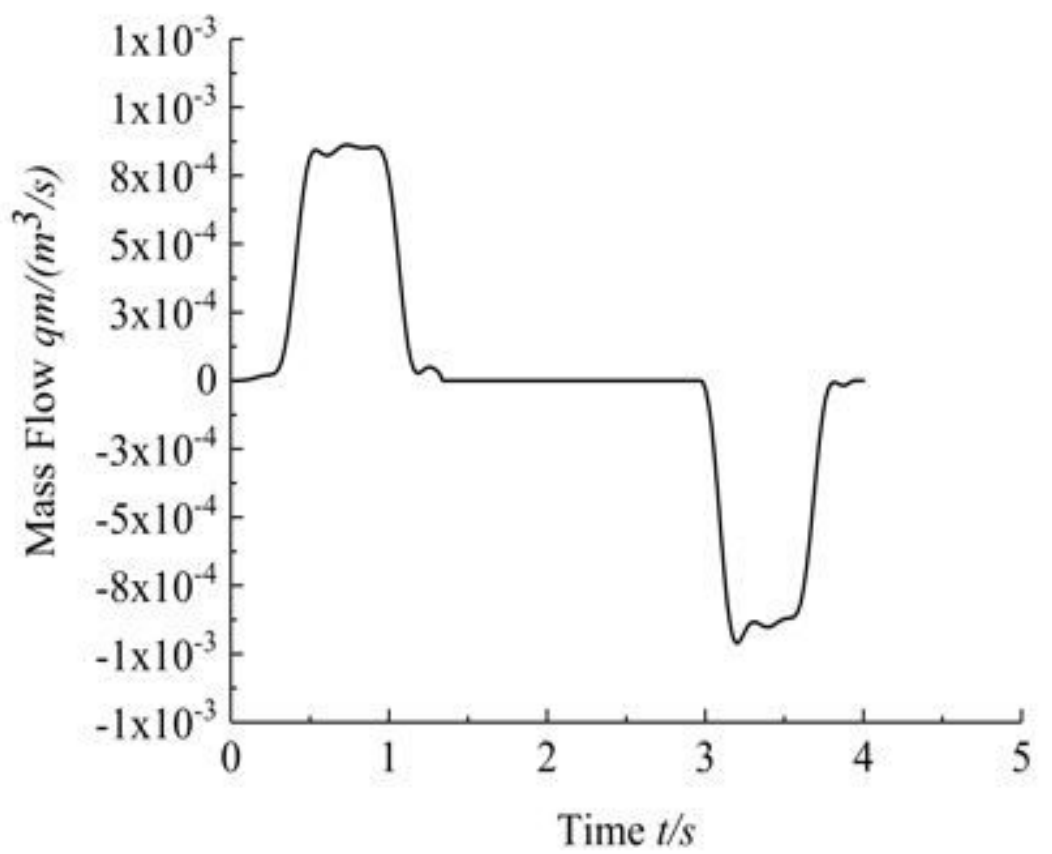

Figure 9

Relationship between mass flow and time of a charging/discharging cycle

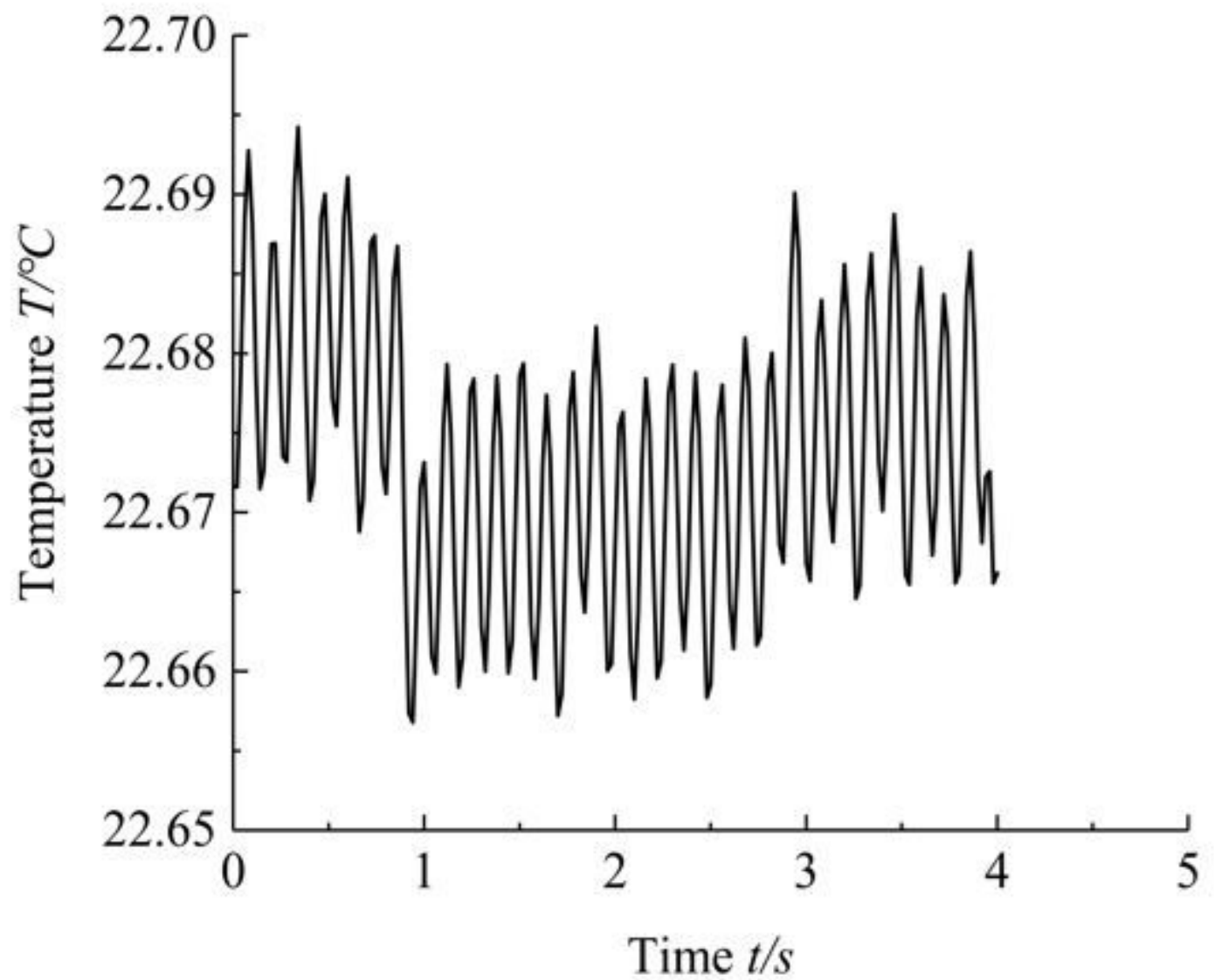


Figure 10

The relationship between temperature and time of a charging/discharging cycle

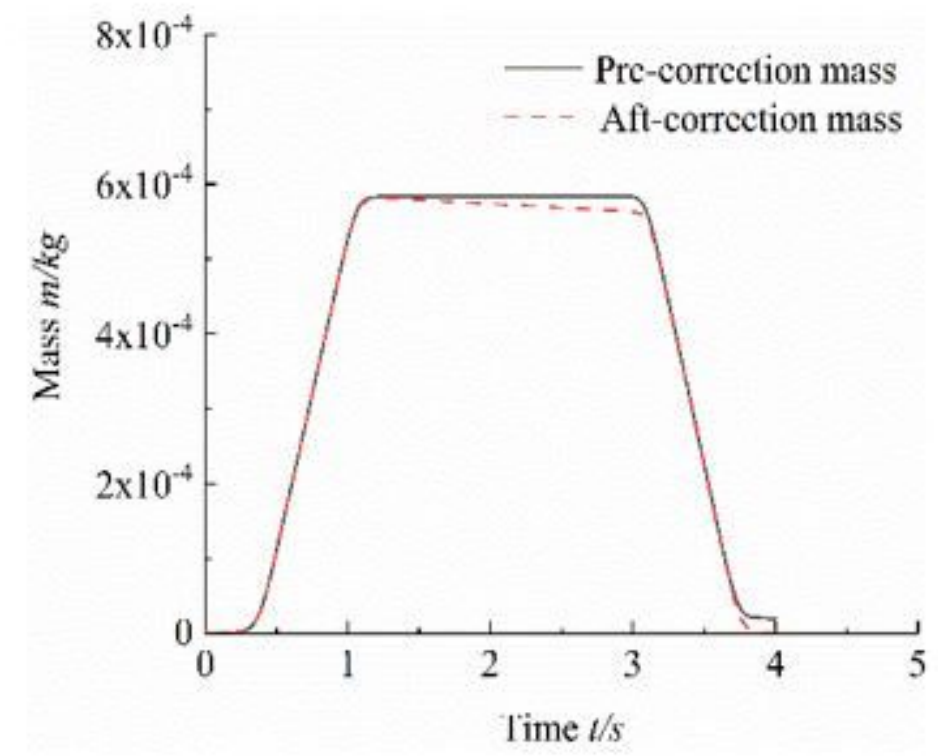

Figure 11

Relationship between gas quality and time before and after adjustment

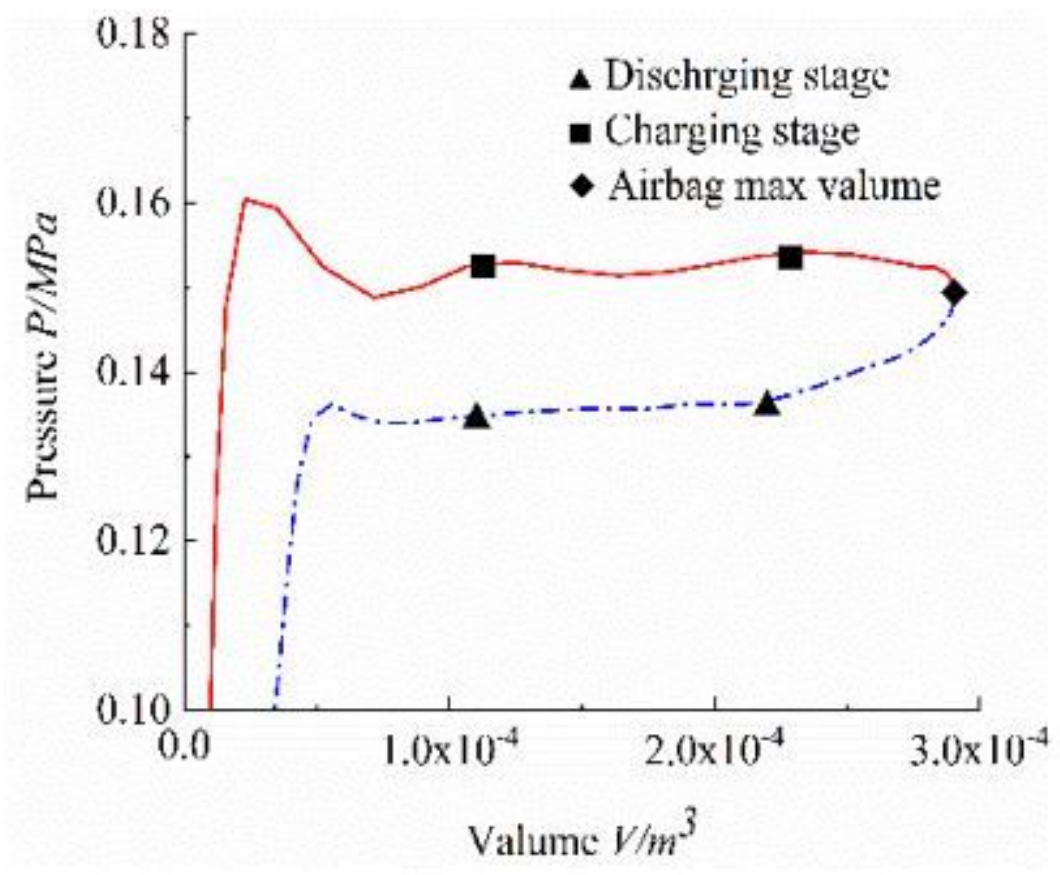

Figure 12

Relationship between gas quality and time before and after adjustment 


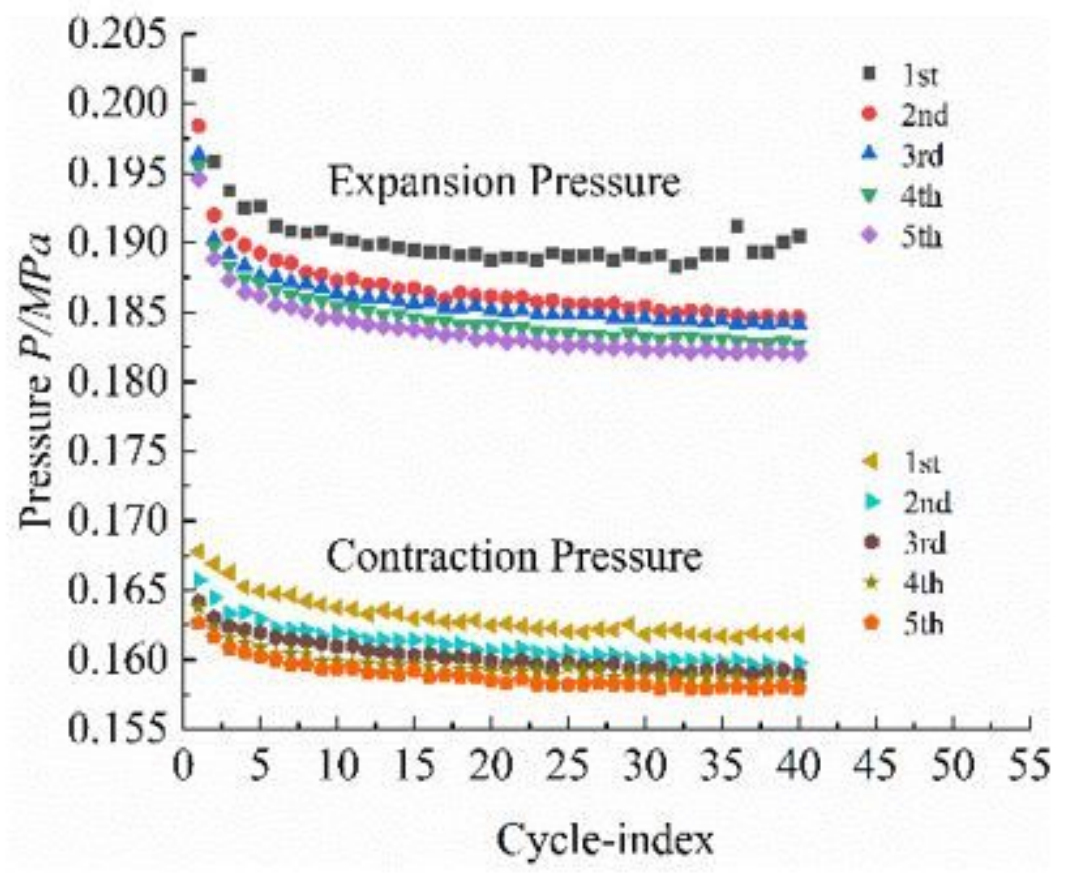

Figure 13

5 test periods changes in expansion pressure and contraction pressure

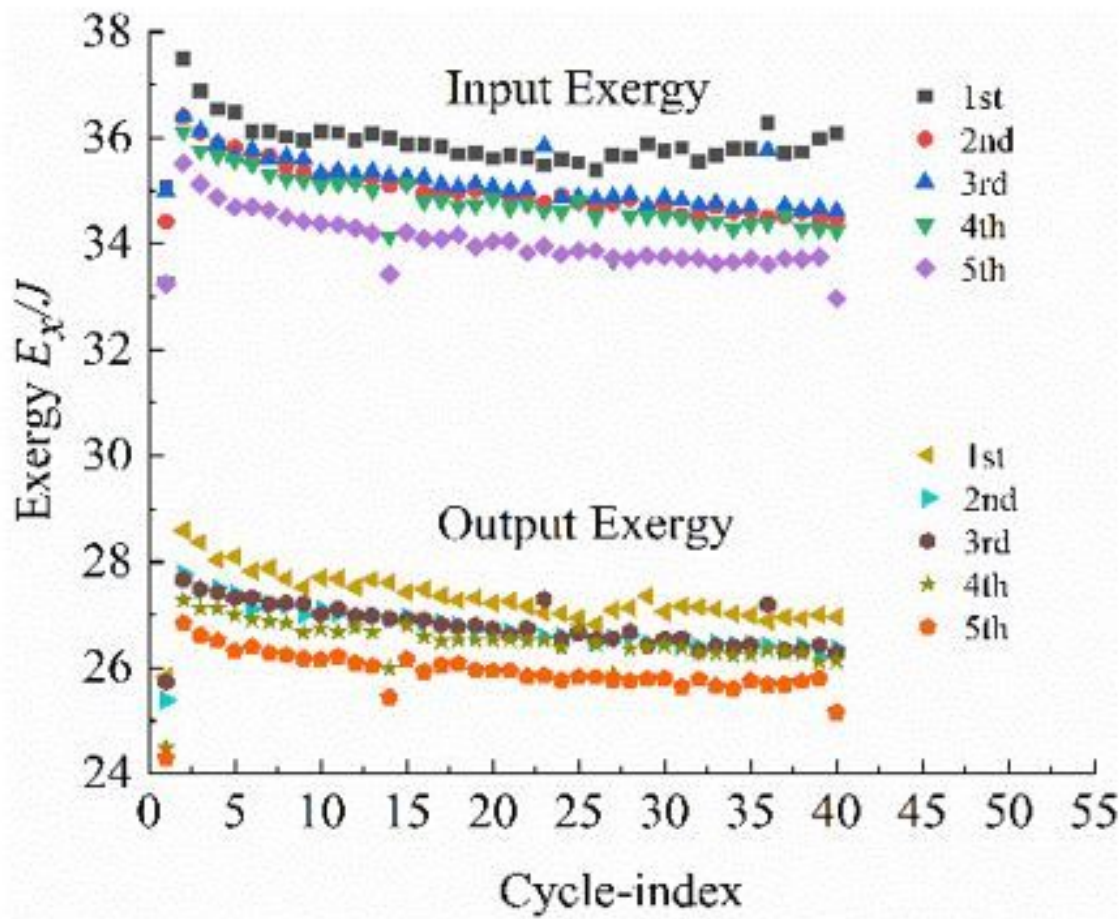

Figure 14

5 test periods in and out of the airbag exergy value 


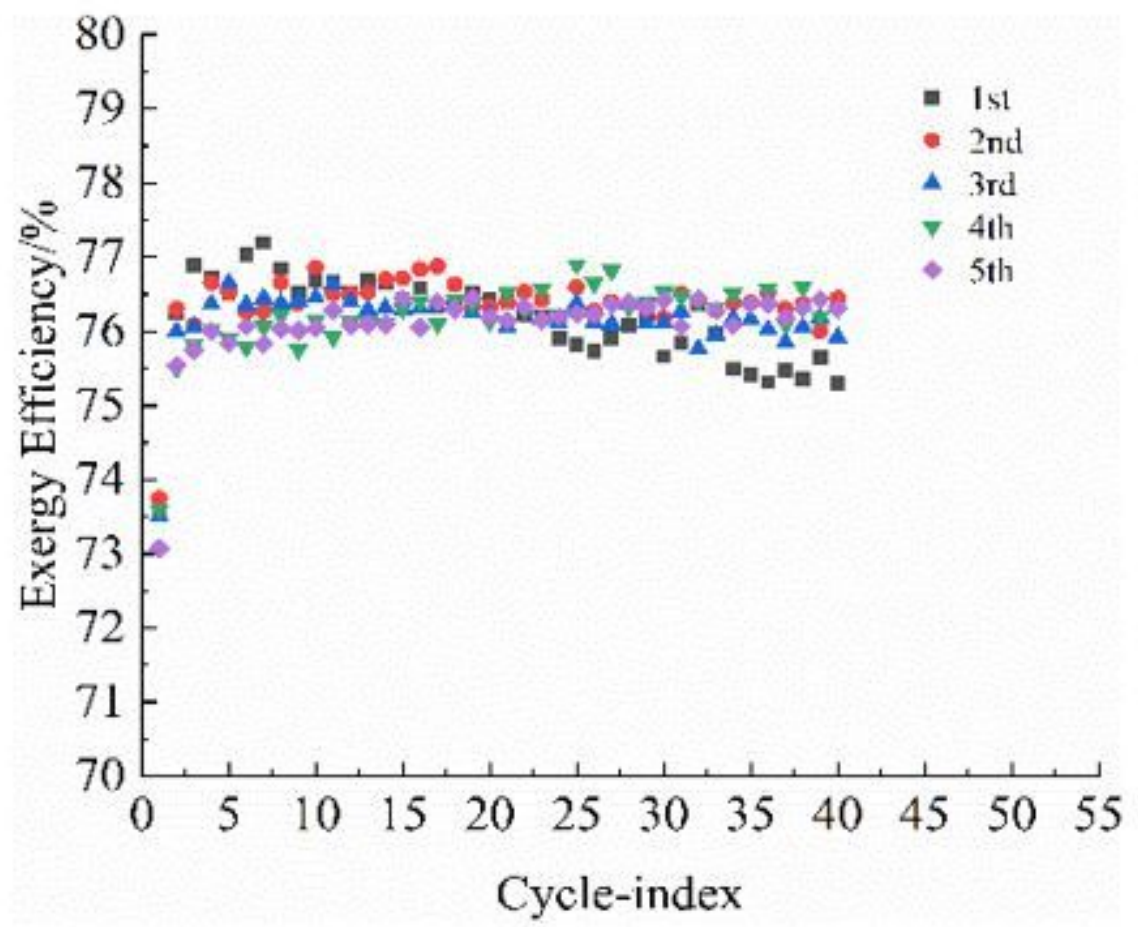

Figure 15

Efficiency changes in 5 charging/discharging periods 